\title{
DARK MATTER IN THE POLAR RING SPIRAL NGC 660
}

\author{
WIM VAN DRIEL \\ Nançay Radio Observatory \\ Observatory of Paris-Meudon, 92195 Meudon, France
}

Polar ring galaxies, where rotation velocities can be measured in two orthogonal planes, are generally considered to be ideal objects for studies of the three-dimensional distribution of dark matter in galaxies.

In the unique, nearby, peculiar polar ring LINER galaxy NGC 660 both the gas-rich polar ring and the equatorial spiral disk were mapped in the $\mathrm{H} \alpha, \mathrm{HI}$, and $\mathrm{CO}(2-1) /(1-0)$ emission lines, as well as in the BVRIJHK bands.

NGC 660 is the only known polar ring galaxy with a gas-rich spiral, rather than a quiescent lenticular, equatorial disk, which allows the measurement of accurate emission-line rotation velocities in both the disk and the polar ring. The polar ring age, estimated from its stellar population, of a few billion years requires a stabilizing mechanism against differential precession. A mass model fit, based on surface photometry and rotation curves, shows that the polar ring is almost as massive as the disk and the dark halo component, indicating self-gravity of the ring as a likely stabilizing mechanism. The three-dimensional shape of the dark halo could not be restrained, however, since there is no overlap in radius between the rotation curves of the equatorial disk and the polar ring; a common, but hitherto unnoted, problem in polar ring galaxy studies.

\section{References}

Combes, F., Braine, J., Casoli, F., Gerin, M. and van Driel, W. (1992), Molecular Gas in a Polar Ring, $A \& A, 259, \mathrm{~L} 65$

van Driel, W., Combes, F., Casoli, F., Gerin, M., Nakai, N., Miyaji, T., Hamabe, M., Sofue, Y., Ichikawa, T., Yoshida, S., Kobayashi, Y., Arimoto, N., Geng, F., Kodama, T., Minezaki, T. Goudfrooij, P., Mulder, P. S., Wakamatsu, K. and Yanagisawa, K. (1994), The Polar Ring Spiral Galaxy NGC 660, A.J., in press 